\title{
Region-based Matching for Robust 3D Face Recognition
}

\author{
A. S. Mian, M. Bennamoun and R. A. Owens \\ School of Computer Science and Software Engineering \\ The University of Western Australia \\ 35 Stirling Highway, Crawley, WA 6009, Australia \\ \{ajmal, bennamou, robyn\}@csse.uwa.edu.au
}

\begin{abstract}
We present a novel region-based matching approach for automatic 3D face recognition which is robust to facial expressions, facial hair, illumination changes and large occlusions. Each 3D face in the gallery is segmented offline into three disjoint regions, namely eyes-forehead, nose and cheeks. Recognition is performed on the basis of only the eyes-forehead and nose regions to avoid the effects of expressions and artifacts that occur in 3D faces due to a mustache or beard. These two regions of the gallery are matched with a probe using a modified version of the ICP algorithm and their matching scores are fused. The identity of the gallery face which gets the highest score is declared as the identity of the probe. Experiments were performed on the UND Biometrics Database which is so far the largest known database of 3D faces. We achieved a combined identification rate of $100 \%$ and a maximum verification rate of $99.42 \%$. Our results also show that the eyes-forehead is the most significant region for 3D face recognition with individual identification and verification rates of $97.32 \%$ and $97.25 \%$ respectively.
\end{abstract}

\section{Introduction}

Biometrics are physiological (e.g. fingerprints and face) and behavioral (e.g. voice and gait) characteristics used to determine or verify an individual's identity [4]. Verification is performed by matching an individual's biometric with the template of the claimed identity only. Identification, on the other hand, is performed by matching an individual's biometric with the template of every identity in the database. In this paper, we will use "recognition" as a combined term for verification and identification.

The face is an easily collectible, universal and non-intrusive biometric [15]. It is ideal for surveillance applications where fingerprinting or iris scanning is not possible. Zhao et al. [34] give a detailed survey of 2D face recognition algorithms and categorize them into holistic matching, feature-based (referred to as region-based ${ }^{1}$ in this work) matching and hybrid methods. Holistic methods use the face as a whole for recognition. Examples of this method include the eigenfaces of Turk and Pentland [29] which use the Principal

${ }^{1}$ We call it region-based to differentiate it from features that are extracted by feature extraction algorithms. 
Component Analysis (PCA), the Fisherfaces [2] which use Linear Discriminant Analysis (LDA), methods based on the Independent Component Analysis (ICA) [1], Bayesian methods [23] and Support Vector Machine (SVM) methods [26]. Neural networks [18] have also been used for holistic face recognition. The region-based methods extract regions like the eyes, nose and mouth and then match these for face recognition. These methods are based on the distances/angles between facial regions or their appearances. Examples of this category include [12][27]. The graph matching approach [32] is one of the most successful region-based approaches [34]. Region-based methods can prove useful in case of variations (e.g. illumination and expression) in the images [34]. Hybrid methods use a combination of the first two approaches. One example is combining the eigenfaces, eigeneyes and eigennose [25]. Other examples include the flexible appearance model-based method [17] and [14]. It is believed that the hybrid approach has the potential to achieve the best results [34].

2D face recognition is sensitive to illumination, pose variations, facial expressions [34] and make up. On the other hand, 3D face recognition has the potential for greater recognition accuracy and is capable of overcoming these limitations [7]. A brief survey of 3D face recognition algorithms include the following. Chua et al. [11] extracted point signatures [10] of the rigid parts of the face for expression invariant face recognition. They reported $100 \%$ recognition results but on a small gallery ${ }^{2}$ of 6 subjects. Xu et al. [33] performed automatic 3D face recognition by combining global geometric features with local shape variation and reported $96.1 \%$ and $72.6 \%$ recognition rates when using a gallery of 30 and 120 subjects respectively. Lu et al. [19][20] used feature detection and registration with the ICP [3] algorithm for 3D face recognition. They report a recognition rate of $96.5 \%$ [20] (using a gallery of 18 subjects) with manual feature detection and a recognition rate of $90 \%$ [19] (using a gallery of 10 subjects) with automatic feature detection. Medioni and Waupotitsch [21] also used a variant to the ICP algorithm for 3D face recognition and reported a recognition rate of $98 \%$ on a gallery of 100 subjects.

Multimodal face recognition which combines the 3D shapes of faces and their corresponding texture maps has also been investigated. These approaches generally perform separate matching on the basis of $2 \mathrm{D}$ and $3 \mathrm{D}$ faces and then fuse the results. Chang et al. [9] used a PCA-based approach for separate 2D and 3D face recognition and fused the matching scores. They reported a recognition rate of $93 \%$ and $99 \%$ for 3D and multimodal face recognition respectively. Their gallery ( 275 subjects) is believed to be the largest in 3D or multimodal face recognition experiments. Wang et al. [31] used Gabor Wavelet filters in the 2D domain and the point signatures [10] in the 3D domain and fused the results using SVM. They reported a recognition rate of above $90 \%$ on a gallery of 50 subjects. Bronstein et al. [8] proposed an expression invariant multimodal face recognition algorithm. They assume the facial surface to be isometric and flatten it to remove the expression effects. However, this process equally flattens all facial features irrespective of whether or not they change with facial expressions (e.g. it flattens the nose to some extent). This lowers the discriminating capability [16] of the faces, which is likely to cause deterioration of the recognition rate as the gallery size increases. A detailed survey on 3D and multimodal face recognition is given by Bowyer et al. [7] who conclude that there is still a need for improved sensors, recognition algorithms and experimental methodology.

We propose a novel region-based approach for robust 3D face recognition. The motivation behind our approach comes from three important findings in the 2D face recog-

\footnotetext{
${ }^{2}$ The database of faces is called the "gallery" and the face to be recognized is called the "probe".
} 


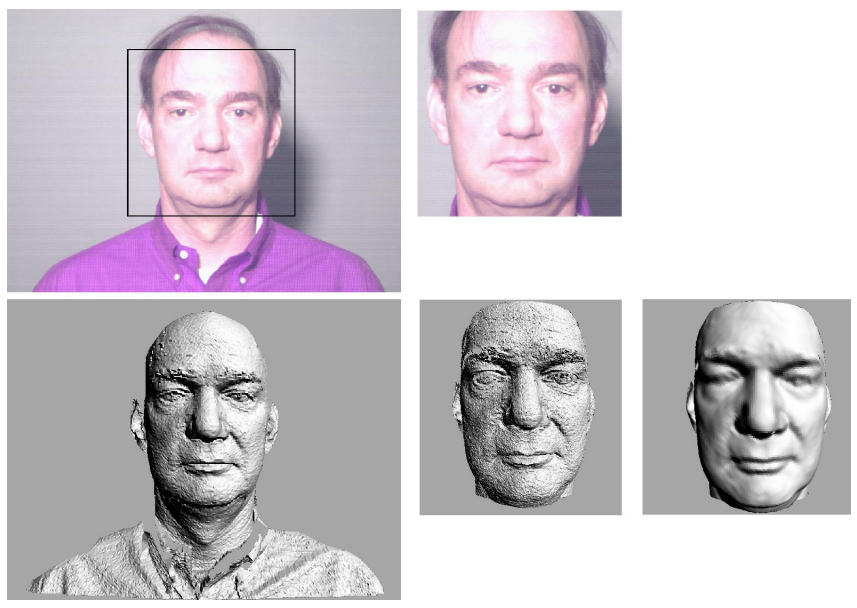

Figure 1: The face area is detected in a 2D image (top left) and cropped. The corresponding area of the face in the $3 \mathrm{D}$ range image (bottom left) is also cropped. The cropped 3D face is then smoothed (column three) to remove the effects of noise.

nition survey of Zhao et al. [34]. One, that the upper part of the face is more significant for recognition compared to the lower part. Two, that region-based matching can prove useful in the case of expression and illumination variations. Three, that the eyes, the forehead and the nose are less sensitive to facial expressions compared to the mouth and the cheeks. Our approach simply extends these ideas to the $3 \mathrm{D}$ face recognition case. We also integrate face and skin detection with our algorithm to develop a fully automatic face recognition system. Note that prior attempts have been made to recognize $3 \mathrm{D}$ faces using regions or segments however these approaches performed a curvature based segmentation of the face (see [7] for details) as opposed to the region-based segmentation in our case. Moreover, the component-based face recognition proposed in [14] performs recognition on the basis of 2D components. Our approach can be considered as multimodal in the sense that it utilizes both 2D and 3D data. However, at present the 2D images are only used for face detection, landmark identification and skin detection. Recognition is performed purely on the basis of $3 \mathrm{D}$ faces. Our aim is to achieve the highest possible recognition rate using only $3 \mathrm{D}$ faces and ultimately fuse our algorithm with a $2 \mathrm{D}$ face recognition algorithm to further improve the recognition performance.

\section{Offline Processing of the Gallery}

The UND 3D face database [9][13] contains multiple frontal snapshots of 277 individuals. Each snapshot includes the 3D range image of an individual's face along with its registered 2D coloured texture map. The resolution of both 2D and 3D images is $480 \times 640$ which is reasonable for 2D images but very high in the 3D case. Moreover, our recognition algorithm does not require high resolution range images. Therefore, we down sampled the $3 \mathrm{D}$ range images (by a factor of $\frac{1}{4}$ ) to $240 \times 320$ by eliminating alternate rows and columns. Moreover, there are noticeable variations in the pose, expression and spatial resolution of the individuals' different snapshots [22] and most of the individuals 


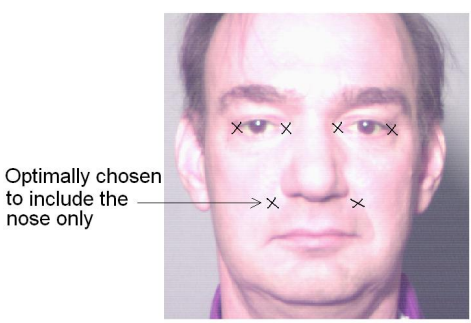

(a)

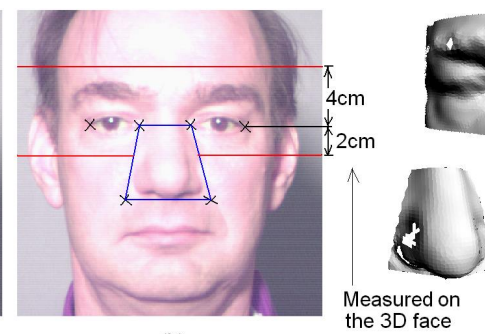

(b)

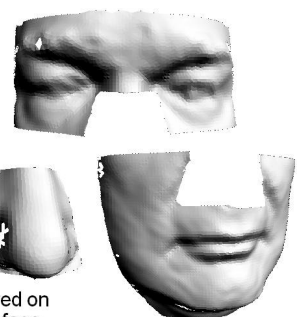

(c)

Figure 2: Six points are manually identified on a 2D face in order to segment the 2D and its corresponding 3D face into three regions i.e. eyes-forehead, nose and cheeks.

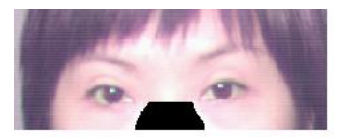

(a)

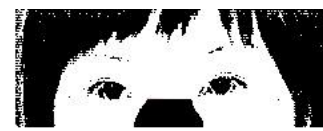

(b)

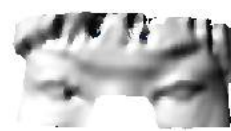

(c)

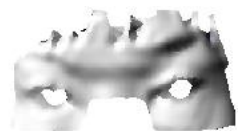

(d)

Figure 3: Skin map (b) generated for the 2D coloured eyes-forehead image (a). The skin map is used to remove non-skin area from the corresponding 3D eyes-forehead ((c) and (d)). Note: Small holes (e.g. in the eyebrows) have been interpolated.

were acquired up to their shoulder level (Fig. 1 first column). Therefore, an important preprocessing requirement was to detect faces in the snapshots. We used Viola and Jones' [30] algorithm for the detection of faces in the 2D images. The detected 2D faces as well as their corresponding 3D faces were then cropped (Fig. 1 second column).

The 3D faces were quite noisy and were therefore smoothed (see Fig. 1 second row) using Taubin's algorithm [28] (50 iterations). Next, the cropped 2D faces were used to manually identify six control points (Fig. 2-a). Note that it is irrelevant whether the identification of these control points is done manually or automatically, as this is performed offline. No manual intervention is required during the online recognition phase. Moreover, the offline manual process can also be replaced with an automatic feature detection algorithm [5]. The identified control points are then used to segment the 2D face (see Fig. 2-b) as well as the corresponding 3D face (Fig. 2-c) into three disjoint regions i.e. the eyes-forehead, the nose and the cheeks. To remove the artifacts in the 3D eyes-forehead region because of hair and eyes [7], we used a skin detection algorithm [6] to detect the skin pixels in the coloured 2D image of the eyes-forehead. The points in the 3D eyesforehead region which did not correspond to the detected skin pixels were removed. Fig. 3 illustrates the skin detection process. In this paper, we only used the eyes-forehead and the nose for region-based 3D face recognition since these regions are the least affected by facial expressions [34] and they also avoid the problems related to a mustache or beard.

\section{Region-based Matching}

During online recognition, the probe face is first detected in the 2D image using the algorithm of Viola and Jones' [30]. The detected face in the 2D image and its corresponding 3D data are then cropped. This is followed by the smoothing [28] of the 3D face and skin detection [6] as described in Section 2. Next, the 3D probe face is matched with the re- 


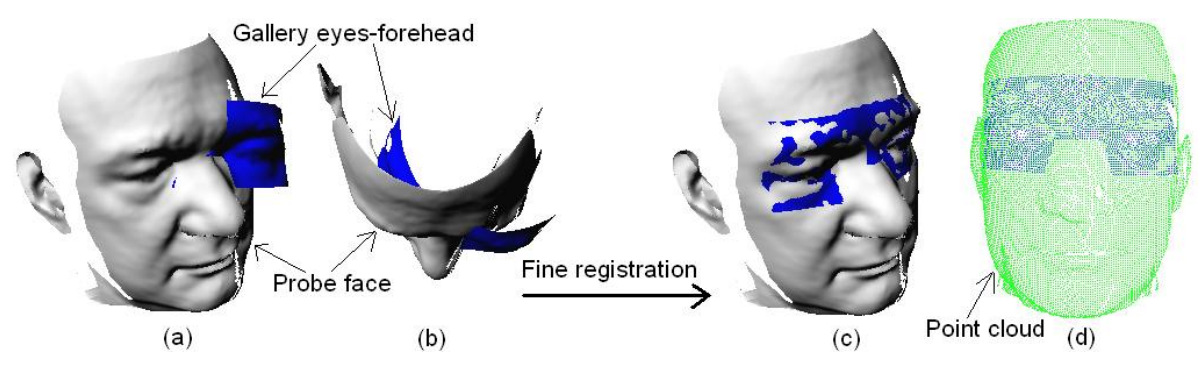

Figure 4: (a)(b) Front and top views of a gallery eyes-forehead registered coarsely to a probe. Prior to registration, the probe was rotated $20^{\circ}$ each about the $\mathrm{x}, \mathrm{y}$ and $\mathrm{z}$ axes. (c)(d) Our modified ICP algorithm successfully converges to a global minimum. Note: Some of the holes have been interpolated.

gions (eyes-forehead and nose) of the gallery faces by registering the gallery regions one by one to the probe. Note that the online recognition process is fully automatic because a prior segmentation of the probe face is not required. Details of the eyes-forehead matching are given in Section 3.1 and that of the nose matching are given in Section 3.2. The individual matching scores are fused (see Section 3.3) to calculate the overall similarity of a gallery face with a probe.

\subsection{Eyes-forehead Matching}

The eyes-forehead of each gallery face is matched by registering it to the probe face. A prior segmentation of the probe face is not required. Registration is performed in two steps. First, the eyes-forehead of the gallery is coarsely registered to the corresponding region of the probe. This initial registration is very coarse and is performed by aligning the centroid of the gallery eyes-forehead to the centroid of the upper two third region of the probe face ${ }^{3}$. This coarse alignment provides an initial estimate which is sufficient for onward refinement with our modified version of the ICP algorithm [3] (explained below in this section) in the second step. Fig. 4-a-b show the initial coarse registration of a gallery eyes-forehead to a probe using the above approach. The coarse registration was performed after rotating the probe by $20^{\circ}$ each about the $\mathrm{x}, \mathrm{y}$ and $\mathrm{z}$ axes. Notice the significant registration error between the two range images. However, the refinement process successfully reduces this error and converges to a global minimum in Fig. 4-c-d. Our registration approach produces reliable results (see Section 4) because of the following reasons. One, our modified ICP algorithm refines the coarse registration hierarchically in order to cater for large initial misalignments. Two, because of its unique structure, it is highly unlikely that the eyes-forehead region will get stuck in a local minimum and therefore it does not require a good initial coarse registration for onward refinement with our modified ICP algorithm. This is in contrast to the case of registering two complete 3D faces (e.g. in the case of [19][20][21]), which may easily get stuck in a local minimum if the initial coarse registration is not accurate.

Our modified ICP algorithm proceeds as follows. First, a high threshold (equal to $4 d_{r}$, where $d_{r}$ is the resolution of the $3 \mathrm{D}$ probe) is chosen for establishing correspondences

\footnotetext{
${ }^{3}$ For more accuracy, the transformation resulting from the nose alignment (Section 3.2) can be used for the coarse registration for the eyes-forehead.
} 
between the two data sets and the registration is iteratively refined till the number of correspondences reaches a maximum saturation value. Only those points of the two data sets which are closer (in 3D) than $4 d_{r}$ are considered corresponding in order to avoid establishing correspondences outside the region of overlap. Next, a more conservative threshold (equal to $2 d_{r}$ ) is chosen for establishing correspondences and the resulting registration is further refined. The stopping criterion is again the maximization of the number of correspondences. At the final stage, the stopping criterion is changed and the iterations stop when the registration error between the two range images stabilizes at a minimum value i.e. there is no significant change in the registration error for a pre-specified number of iterations. Moreover, correspondences are established between all pairs of nearest points except for those whose mutual distance is greater than the threshold $\left(2 d_{r}\right)$ in the xy plane only. Points which are close in the xy plane, but far in the $\mathrm{z}$ dimension ${ }^{4}$, are considered corresponding points. These points provide useful information regarding the dissimilarity between the probe and gallery eyes-forehead. The average registration error $e_{F}$ between the eyes-forehead and the probe is calculated using Eqn. 1.

$$
e_{F}=\frac{1}{n d_{r}} \sum_{i=1}^{n}\left\|\mathbf{G}_{F i} \mathbf{R}+\mathbf{t}-\mathbf{P}_{i}\right\|
$$

In Eqn. 1, $\mathbf{G}_{F i}$ and $\mathbf{P}_{i}$ are the corresponding points of the gallery eyes-forehead and the probe face respectively. $\mathbf{R}$ and $\mathbf{t}$ are the rotation matrix and translation vector that aligns the gallery eyes-forehead to the probe. $n$ is the total number of corresponding points and $d_{r}$ is the resolution of the probe.

\subsection{Nose Matching}

The nose of each gallery face is matched by registering it to the probe nose. As in the case of the eyes-forehead, a prior segmentation of the nose region of the probe is not necessary. The registration in this case is also performed in two steps. First, the gallery nose is coarsely registered to the probe. Unlike the eyes-forehead region, the nose registration can easily get stuck in a local minimum in case the initial coarse registration is not accurate enough. Therefore, we used a more robust approach for the coarse registration of the gallery nose to the probe. We detected the ridge line [24] and the point of maximum slope of the gallery and probe noses and aligned them. The nose ridge is detected as follows. The probe face and the gallery nose are sliced horizontally at varying steps and a cubic spline is fitted into each set of sliced points (see Fig. 5-a). A spline is used so that the peak of each slice can be accurately detected even if it lies in between two data points. Next, a line is passed through the peak points of all the slices using RANSAC. This line forms the nose ridge line as shown in Fig. 5-b. In the case of the probe, every slice may not contain the nose. Therefore, a decision is made based upon the curvatures of the spline, the length of sides and area of the triangle (Fig. 5-a) on whether or not the slice contains the nose. The point of maximum slope of each nose is detected as follows. The probe is vertically sliced at its detected ridge line, a cubic spline is fitted into the sliced points and the point of maximum slope is then detected in the spline (see Fig. 5-c).

Once the gallery nose is coarsely registered to the probe, the registration is further refined with our modified ICP algorithm described in Section 3.1. However, in this case,

\footnotetext{
${ }^{4}$ The subjects are facing the positive $\mathrm{z}$-axis.
} 


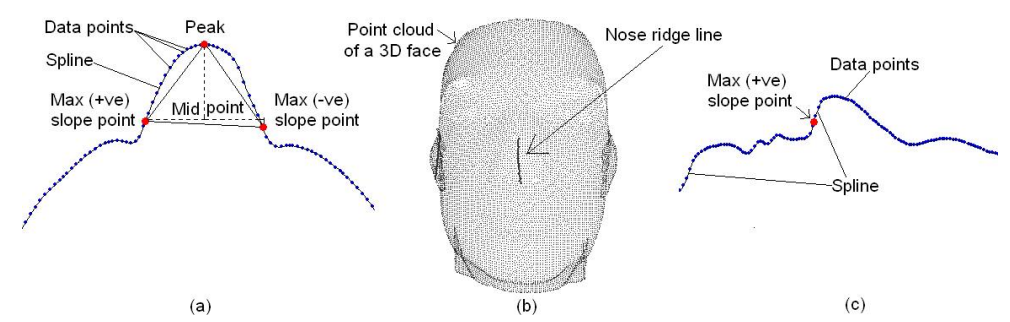

Figure 5: (a) A horizontal slice of a 3D face. The data points are interpolated with a cubic spline. The points of maximum slopes are detected and their midpoint is used to detect the peak of the spline. (b) A line is passed through the detected peak points using RANSAC to find the nose ridge of the 3D face (shown as point cloud). (c) The face is sliced at the ridge line to detect the point of maximum slope.

correspondences at the final stage are established in a similar way to the initial two stages. The average error $e_{N}$ between a gallery nose and a probe is calculated using the same Eqn. 1 (replacing $e_{F}$ by $e_{N}$ and $\mathbf{G}_{F i}$ by $\mathbf{G}_{N i}$ ).

\subsection{Fusion}

The errors $e_{F}$ and $e_{N}$ of each gallery face are fused using Eqn. 2 to calculate its overall similarity $S$ with the probe. The identity of the gallery face with the highest value of $S$ is declared as the identity of the probe. We tested three different fusion rules namely, sum, weighted sum and product and observed that the product rule (Eqn. 2) produces the best recognition results.

$$
S=\frac{1}{e_{N} e_{F}}
$$

\section{Results}

We performed our experiments on the UND 3D face database [9][13] comprising 2D/3D images of 277 subjects. The size of the gallery was 277 and the number of tested probes was 671. A single snapshot (2D and 3D face) per individual was used in the gallery. Recognition was also performed on the basis of matching a single snap shot of a probe with the gallery. Fig. 6 shows our identification results in the form of rank identification rate on the basis of individual and combined regions. Note that individually, the eyesforehead performed much better than the nose with a rank one identification rate of $97.3 \%$ compared to $81.8 \%$ in the case of the nose. At rank eight, the eyes-forehead reached $100 \%$ and the nose reached $98 \%$ identification rate. After fusion, a combined rank one identification rate of $100 \%$ was acheived. This can be compared with the recognition rate of $93 \%$ acheived by Chang et al. [9] on the same database when using a holistic 3D matching approach. Our recognition rates are reported assuming $100 \%$ face detection. The automatic face detection [30] rate in our experiments was $99.2 \%$. The remaining $0.8 \%$ faces were manually detected.

The above identification experiments amounted to $170909(671 \times 277)$ verification trials. Out of these, 671 were genuine (a probe was matched with its correct identity in the 


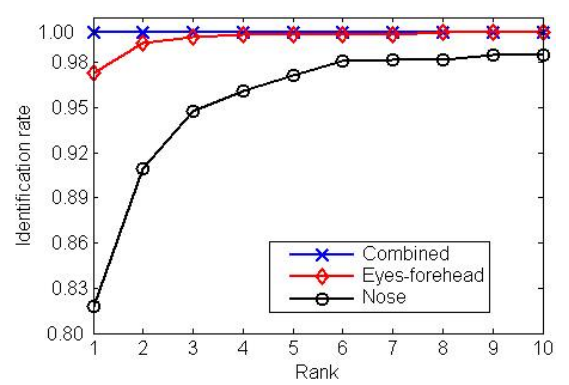

Figure 6: Identification results.

gallery) and the remaining 170238 were impostors (a probe was matched with an identity in the gallery other than its own). The verification performance of our algorithm was analyzed by calculating the genuine and impostor probability distributions (Fig. 7 first row) and plotting the ROC (Receiver Operating Characteristic) curves (Fig. 7 second row) for the individual and combined matching results. Notice that the overlap between the genuine and impostor distributions gets smaller as we go from the nose to the combined scores. Moreover, the distance between the two probability distributions in the combined case is much greater than the individual cases. The ROC curves (Fig. 7 second row) show the false acceptance rates versus the false rejection rates. These rates vary with the selected threshold for verification. For comparison, we selected the threshold at which the sum of these two errors becomes minimum for a given ROC curve. With this criterion, the error rates (false acceptance + false rejection) were $6.91 \%, 2.75 \%$ and $0.58 \%$ for the nose, eyes-forehead and the combined case respectively.

Note that the above experiments were performed after down sampling the 3D faces of the UND database by a factor of $\frac{1}{4}$ which shows a strength of our algorithm that it does not require high resolution scanners. Another important advantage of our region-based matching approach is that it can operate under large occlusions. In our experiments, the eyes-forehead region covered only $22.4 \%$ and the nose covered only $7.5 \%$ of the area of a face on average. Therefore, a probe face which is $77.6 \%$ occluded and whose eyes-forehead region is only visible can still be recognized by our algorithm with $97.3 \%$ accuracy. This is of great significance in surveillance applications where the complete face may not always be visible to the sensor.

\section{Summary and Conclusions}

We presented a novel region-based matching approach for robust 3D face recognition. Our algorithm is fully automatic and does not require any manual interaction during the online recognition process. Unlike most existing published work in the area of 3D face recognition, we performed our experiments using the largest available database of 3D faces. We achieved $100 \%$ recognition rate by matching only the 3D eyes-forehead and nose regions which is a crucial accomplishment in the area of expression invariant face recognition. In the future we intend to utilize the remaining information contained in the $2 \mathrm{D}$ images and the cheeks region using non-rigid matching techniques in order to further improve the verification performance. 

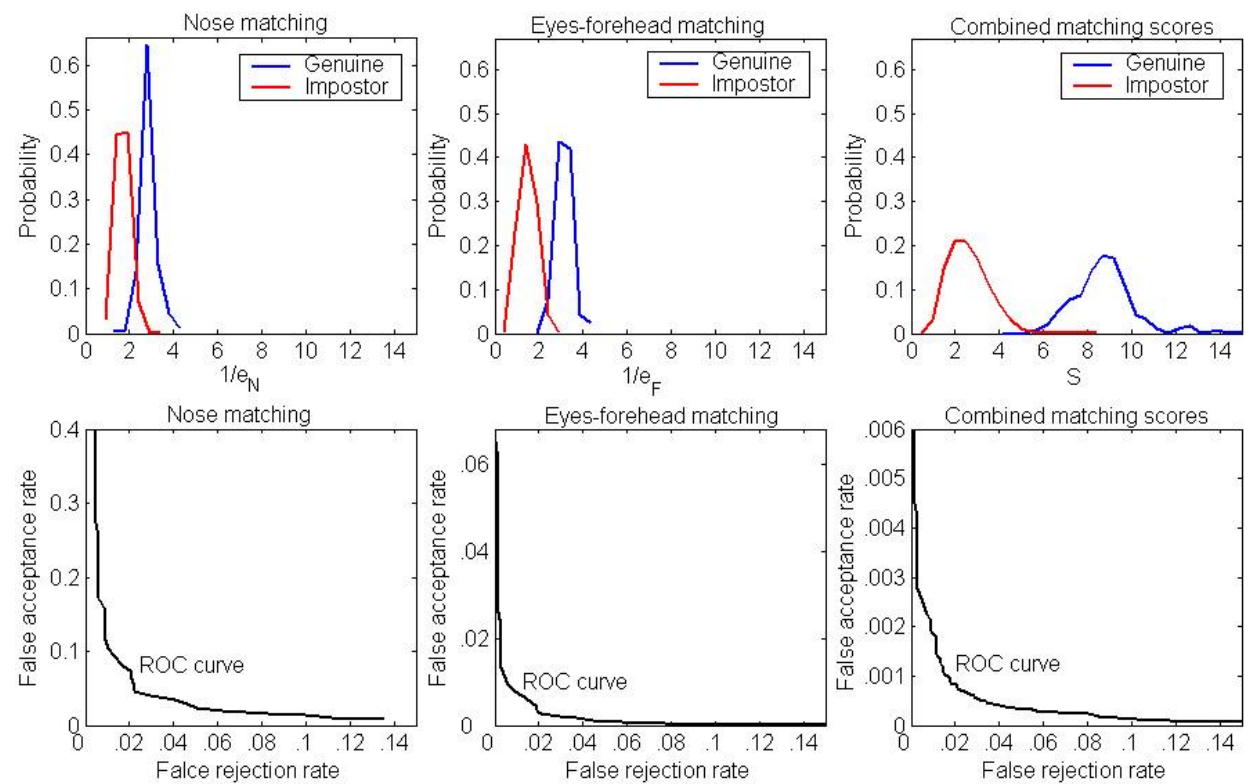

Figure 7: Verification results. Notice the difference in the vertical scales between the graphs of the second row.

\section{Acknowledgements}

Thanks to UND for the face data, CMU for the Mesh Toolbox and F. Boussaid for the skin detection code. This research is supported by ARC grant DP0344338.

\section{References}

[1] M. S. Bartlett, H. M. Lades and T. Sejnowski, "Independent Component Representation for Face Recognition", SPIE, pp. 528-539, 1998.

[2] P. Belhumeur, J. Hespanha and D. Kriegman, "Eigenfaces vs. Fisherfaces: Recognition Using Class Specific Linear Projection”, IEEE PAMI, vol. 19, pp. 711-720, 1997.

[3] P. J. Besl and N. D. McKay, "Reconstruction of Real-world Objects via Simultaneous Registration and Robust Combination of Multiple Range Images," IEEE TPAMI, vol. 14(2), pp. 239-256, 1992.

[4] Biometric Consortium, http://www.biometrics.org, 2004.

[5] C. Boehnen and T. Russ, "A Fast Multi-Modal Approach to Facial Feature Detection", IEEE WACV, 2005.

[6] F. Boussaid, D. Chai and A. Bouzerdoum, "A Current-mode VLSI Architecture for Skin Detection”, ISSPA, vol. 1, pp. $629-632,2003$.

[7] K. W. Bowyer, K. Chang and P. Flynn, "A Survey Of Approaches to ThreeDimensional Face Recognition,” IEEE ICPR, pp. 358-361, 2004.

[8] A. M. Bronstein, M. M. Bronstein and R. Kimmel, "Expression-invariant 3D Face Recognition," AVBPA pp. 62-70, 2003.

[9] K. I. Chang, K. W. Bowyer and P. J. Flynn, "Face Recognition Using 2D and 3D Facial Data," MMUA, pp. 25-32, 2003.

[10] C. S. Chua and R. Jarvis, "Point Signatures: A New Representation for 3D Object Recognition," IJCV, vol. 25(1), pp. 63-85, 1997. 
[11] C. Chua, F. Han and Y. Ho, "3D Human Face Recognition Using Point Signatures," IEEE AMFG, pp. 233-238, 2000.

[12] I. J. Cox, J. Ghosn and P. N. Yianilos, "Feature-based Face Recognition using Mixture Distance", IEEE CVPR, pp. 209-216, 1996.

[13] P. J. Flynn, K. W. Bowyer and P. J. Phillips, "Assessment of time dependency in face recognition: An initial study", AVBPA, pp. 44-51, 2003.

[14] J. Huang, B. Heisele and V. Blanz, "Component-based Face Recognition with 3D Morphable Models", AVBPA, 2003.

[15] A. K. Jain, L. Hong and S. Pankanti, "Biometric Identification," Communications of the ACM, vol. 43(2), pp. 91-98, 2000.

[16] A. K. Jain, A. Ross and S. Prabhakar, "An Introduction to Biometric Recognition," IEEE TCSVT, vol. 14(1), pp. 4-20, 2004.

[17] A. Lanitis, C. Taylor and T. Cootes, "Automatic Face Identification System Using Flexible Appearance Models", Image \& Vision Comp., Vol. 13, pp. 393-401, 1995.

[18] C. Liu and H. Wechsler, "Evolutionary Pursuit and its Application to Face Recognition", IEEE TPAMI, Vol. 22, pp. 570-582, 2000.

[19] X. Lu, D. Colbry and A. K. Jain, "Matching 2.5D Scans for Face Recognition," ICBA, LNCS 3072, pp. 30-36, 2004.

[20] X. Lu, D. Colbry and A. K. Jain, "Three-dimensional Model Based Face Recognition," IEEE ICPR, 2004.

[21] G. Medioni and R. Waupotitsch, "Face Recognition and Modeling in 3D", IEEE $A M F G$, pp. 232-233, 2003.

[22] A. S. Mian, M. Bennamoun and R. A. Owens, "Matching Tensors for Pose Invariant Automatic 3D Face Recognition", A3DISS, 2005.

[23] B. Moghaddam and A. Pentland, "Probabilistic Visual Learning for Object Representation”, IEEE TPAMI, Vol. 19, pp. 696-710, 1997.

[24] D. T. J. O’Mara, “Automated Facial Metrology,” PhD Thesis, Computer Science \& Software Engg., The University of Western Australia, 2002.

[25] A. Pentland, B. Moghaddam and T. Starner, "View-based and Modular Eigenspaces for Face Recognition”, IEEE CVPR, 1994.

[26] P. J. Phillips, "Support Vector Machines Applied to Face Recognition", Adv. Neural Inform. Process. Syst., Vol. 11, pp. 803-809, 1998.

[27] F. Samaria and S. Young, "HMM based Architecture for Face Identification", International Journal of Image and Vision Computing, Vol. 12, pp. 537-583, 1994.

[28] G. Taubin, "Curve and Surface Smoothing without Shrinkage," ICCV, pp. 852-857, 1995.

[29] M. Turk and A. Pentland, "Eigenfaces for Recognition”, JOCN, Vol. 3, 1991.

[30] P. Viola and M. J. Jones, "Robust Real-Time Face Detection”, IJCV, Vol. 57(2), pp. 137-154, 2004.

[31] Y. Wang, C. Chua and Y. Ho, "Face Recognition From 2D And 3D Images," AVBPA, pp. 26-31, 2001.

[32] L. Wiskott, J. Fellous and C. Malsburg, "Face Recognition by Elastic Bunch Graph Matching”, IEEE TPAMI, Vol. 19, pp. 775-779, 1997.

[33] C. Xu, Y. Wang, T. Tan and L. Quan, "Automatic 3D Face Recognition Combining Global Geometric Features with Local Shape Variation Information," IEEE ICPR, pp. 308-313, 2004.

[34] W. Zhao, R. Chellappa, P.J. Phillips, and A. Rosenfeld, "Face Recognition: A Literature Survey", ACM Computing Survey, pp. 399-458, 2003. 